\title{
UMA PROPOSTA PARA A MENSURAÇÃO DO AUTOCUIDADO EM IDOSOS
}

Selma Petra Chaves Sá1, Dirley Moreira dos Santos², Lorena Maria Volkers Robers ${ }^{3}$, Marcia Santos Andrade ${ }^{4}$, Carlos Alberto Quadros Coimbra ${ }^{5}$, Thiara Joanna Peçanha da Cruz ${ }^{6}$

RESUMO: Estudo quantitativo objetivou construir escalas de Mokken relacionadas à capacidade de autocuidado de 48 idosos. A pesquisa utilizou a versão validada para o português do instrumento Appraisal of Self-Care Agency Scale (ASA-A) para avaliação do autocuidado. O questionário com 24 itens admite respostas em uma escala Likert com cinco alternativas. Para a análise, utilizou-se a teoria da resposta ao item não paramétrica. Do total de itens politômicos, 19 formaram duas escalas de Mokken: a de Capacidade Subjetiva de Autocuidado, com 11 itens e a de Capacidade Objetiva de Autocuidado, com 8 itens; Foi possível ordenar em duas escalas, de maior a menor capacidade de autocuidado, segundo o escore total de cada uma. Verificou-se que quando se trata de ações e atitudes objetivas, a capacidade de autocuidado dos idosos é menor do que quando se trata de ações e atitudes subjetivas.

PALAVRAS-CHAVE: Autocuidado; Idoso; Enfermagem gerontológica.

\section{A PROPOSAL FOR THE MEASURING OF SELF-CARE IN THE ELDERLY}

ABSTRACT: This quantitative study aimed to construct Mokken scales related to the capacity for self-care of 48 elderly individuals. The survey used a version of the Appraisal of Self-Care Agency Scale validated for Portuguese. The questionnaire of 24 items permitted answers on a Likert scale with five alternatives. For analysis, non-parametric item response theory was used. Out of all the polytomic items, 19 formed two Mokken scales: that of Subjective Self-Care Capacity, with 11 items, and that of Objective Self-Care Capacity, with 8 items. It was possible to set up two scales in diminishing order of self-care ability, according to each person's total score. It was verified that where objective actions and attitudes are concerned, the self-care capacity of the elderly is less than when one is considering subjective actions and attitudes.

KEYWORDS: Self-care; Elderly; Gerontological care.

\section{UNA PROPUESTA PARA LA MENSURACIÓN DEL AUTOCUIDADO EN ANCIANOS}

RESUMEN: Estudio cuantitativo cuyo objetivo fue construir escalas de Mokken relacionadas a la capacidad de autocuidado de 48 ancianos. La investigación ha utilizado la versión del portugués del instrumento Appraisal of Self-Care Agency Scale (ASA-A) para evaluación del autocuidado. El cuestionario con 24 ítems puede tener respuestas en una escala Likert con cinco alternativas. Para el análisis, se utilizó la teoría de la respuesta al ítem no paramétrica. Del total de ítems politómicos, 19 formaron dos escalas de Mokken: la de Capacidad Subjetiva de Autocuidado, con 11 ítems y la de Capacidad Objetiva de Autocuidado, con 8 ítems. Fue posible ordenar en dos escalas, de mayor a menor capacidad de autocuidado, de acuerdo con el score total de cada una. Se ha verificado que, cuando se trata de acciones y actitudes objetivas, la capacidad de autocuidado de los ancianos es menor que cuando se trata de acciones y actitudes subjetivas.

PALABRAS-CLAVE: Autocuidado; Anciano; Enfermería gerontológica.

${ }^{1}$ Enfermeira. Doutora em Enfermagem. Professora do Curso de Graduação e do Programa de Pós-Graduação da Escola de Enfermagem Aurora de Afonso Costa da Universidade Federal Fluminense - UFF.

${ }^{2}$ Estatística. Doutora em Estatística. Professora do Instituto de Matemática e Estatística da UFF.

${ }^{3}$ Enfermeira.

${ }^{4}$ Graduada em Matemática. Mestre em Matemática. Doutoranda do Programa de Pós-Graduação pela Pontíficia Universidade Católica do Rio de Janeiro.

${ }^{5}$ Engenheiro. Doutor em Engenharia Elétrica. Estatístico do Museu de Astronomia e Ciências Afins do Rio de Janeiro.

${ }^{6}$ Enfermeira. Mestranda do Programa de Pós-Graduação em Ciências do Cuidado em Saúde pela UFF. 


\section{INTRODUÇÃO}

A questão do envelhecimento vem sendo objeto de preocupação em países em desenvolvimento, inclusive no Brasil. O aumento da expectativa de vida ou longevidade vem acompanhado de problemas de saúde de longa duração, implicando em custos, tanto para o indivíduo quanto para a sociedade de modo geral. Estima-se que, no Brasil, este contingente se elevará de tal forma que o país ocupará uma posição de destaque, em 2025, em relação a outros países do mundo(1).

Da sua concepção até a morte, o ser humano passa pelo nascimento, puberdade, maturidade e chega ao envelhecimento. A capacidade de cuidar de si pode estar afetada diante de uma doença, em qualquer fase da vida; entretanto, tal capacidade pode se apresentar mais comprometida no idoso. E é na última fase da vida que há o declínio das funções vitais que podem comprometer o cuidado de si. Para Orem, o autocuidado é a capacidade que o indivíduo tem de cuidar de $\mathrm{si}^{(2)}$ e, para o idoso, o desempenho nas Atividades da Vida Diária é parâmetro amplamente aceito e reconhecido, pois pode demonstrar sua capacidade funcional, o que contribui para que os profissionais tenham uma visão mais precisa quanto à gravidade das doenças, suas sequelas e do cuidado a ser prestado.

A capacidade de envolver-se e executar ações para o autocuidado são aprendidas e estão sujeitas a alguns fatores condicionantes como idade, experiências de vida, valores, cultura, recursos financeiros, sexo e nível educacional. $\mathrm{O}$ autocuidado é comprometido quando o idoso apresenta dificuldade no desempenho das Atividades de Vida Diária, não atendendo de forma eficaz suas necessidades diárias de alimentação, higiene, bem-estar psicológico e lazer.

A capacidade funcional tem uma estreita relação com o autocuidado. Sabe-se da importância, para o idoso, de que os profissionais se assegurem das reais possibilidades de déficit de autocuidado nesta fase da vida. Mesmo que não haja déficit, o profissional de saúde, entre eles o enfermeiro, pode atuar detectando os riscos, além de promover ações para a manutenção do cuidado de si do idoso. Contudo, o autocuidado pode ter características subjetivas e objetivas. Neste artigo, considera-se como subjetividade o mundo interno de todo e qualquer ser humano, composto por emoções, sentimentos e pensamentos; e como objetividade, a qualidade daquilo que é objetivo, externo, resultado de observação imparcial, independente das preferências individuais ${ }^{(3)}$. Assim, no autocuidado subjetivo ocor- rem ações que emergem das emoções e sentimentos, e o objetivo são as condutas e atitudes que os idosos realizam para o cuidado de si.

Em escalas para avaliação do autocuidado, vê-se a preocupação de levantar diferentes considerações e/ou percepções dos sujeitos, tendo em vista sua subjetividade e sua objetividade. Considera-se de suma importância a avaliação do autocuidado desenvolvida para idosos utilizando instrumentos específicos para este fim. Neste estudo, optou-se pela versão em português, validada, do instrumento de autocuidado Appraisal of Self-Care Agency Scale (ASA-A) ${ }^{(4-5)}$.

O questionário ASA-A não divide os seus 24 itens em autocuidado subjetivo e objetivo. Considerando a importância dessa separação para a atuação dos profissionais de saúde, na análise de natureza quantitativa, utilizou-se a Teoria da Resposta ao Item Não Paramétrica (TRIN), por meio do ajuste do modelo de Mokken $^{(6)}$, através do programa MSP (Mokken Scale for Politomous items) $)^{(7)} \mathrm{em}$ seu modo confirmatório. O seu emprego teve por objetivo verificar se a divisão proposta apresenta relevância e pode ser considerada ao se aplicar o referido questionário.

A presente pesquisa teve por objetivo construir escalas de Mokken relacionadas à capacidade de autocuidado de idosos com doenças crônicas.

\section{METODOLOGIA}

Esta pesquisa quantitativa foi realizada no período de 2008 e com uma amostra não probabilística de 48 idosos com doenças crônicas inscritos no programa "A Enfermagem na Assistência à Saúde do Idoso e seus Cuidadores" da Universidade Federal Fluminense (EASIC/UFF). Utilizaram-se como critérios de inclusão: estar inscrito no EASIC/UFF e ter 60 anos ou mais com até duas doenças crônicas diagnosticadas; e como critério de exclusão, aqueles com qualquer tipo de demência em vista da potencial dificuldade cognitiva desses em responder as questões do estudo. Foram observados os procedimentos éticos e a pesquisa foi aprovada pelo Comitê de Ética da UFF sob o n. 111/07.

A pesquisa para aplicação do formulário ASAA em idosos foi realizada através de entrevista com perguntas fechadas contidas no referido instrumento. O questionário abrange 24 itens que, de modo geral, dizem respeito à disponibilidade, vontade e condições dos idosos em modificar as suas vidas, ou seja, dados mais subjetivos. Também se investigou com os cuidados com a alimentação, higiene e peso, dados 
mais objetivos do autocuidado. Estes 24 itens admitem respostas em uma escala de Likert com cinco alternativas: discordo totalmente, discordo, nem concordo nem discordo, concordo e concordo totalmente.

Com o intuito de possibilitar melhor caracterização dos respondentes com relação a fatores sociais, foram consideradas as seguintes variáveis categóricas: sexo, idade (60 a 69, 70 a 79, acima de 80 anos), escolaridade (ensino fundamental incompleto, fundamental completo, ensino médio incompleto, médio completo e ensino superior) e estado civil (solteiro, casado, viúvo, separado e outro).

A Teoria de Resposta ao Item (TRI) desempenha um papel muito importante, apresentando vários modelos matemáticos para a construção de escalas em diversas áreas do conhecimento. A TRI propõe uma versão estocástica da escala determinística de Guttman, denominada de Teoria de Resposta ao Item Não Paramétrica (TRIN). Em ambas as versões, a curva de resposta ao item descreve a probabilidade de ocorrer determinada resposta dado o traço latente do indivíduo. Assim, uma escala perfeita, determinística, chamada escala de Guttman, é aquela em que a posição do indivíduo está determinada com exatidão, entre dois itens ${ }^{(8)}$, neste caso, para a objetividade e a subjetividade no autocuidado do idoso. É o caso da escala formada por itens como "consigo ir ao toalete sozinho", "consigo fazer compras sozinho" e "consigo andar dois quilômetros"; quem responde sim a um dos itens, certamente responderá sim aos anteriores.

Mokken desenvolveu três coeficientes de escalonabilidade com o objetivo de determinar o ajuste do modelo $\mathrm{MH}$ aos dados. Estes coeficientes estão baseados no suposto de independência marginal. $\mathrm{O}$ primeiro deles, $\mathrm{H}_{\mathrm{ij}}$, mede o grau de associação entre cada par de itens $i \mathrm{e} j$. O segundo, $\mathrm{H}_{\mathrm{i}}$, mede a homogeneidade de um item particular, referente a um conjunto de itens. $\mathrm{O}$ terceiro, denominado $\mathrm{H}$ de Loevinger, ou coeficiente de escalonabilidade, mede a homogeneidade da escala como um todo ${ }^{(6)}$.

A escala de Guttman proposta na TRI tem um coeficiente de escalonabilidade igual a um. À medida que a escala de Mokken se afasta do padrão de Guttman, a escalonabilidade $\mathrm{H}$ diminui. Um valor de $\mathrm{H}$ igual a zero indica que os itens não formam escala alguma. Neste trabalho, como o objetivo foi construir escalas relacionadas à capacidade de autocuidado objetivo e subjetivo, o $\mathrm{H}$, necessariamente, tem que estar acima do valor zero para que se formem tais escalas.

$\mathrm{Na}$ análise de Mokken são apresentados os níveis aceitáveis de $\mathrm{H}$ para a qualidade da escala. Isto tem estreita relação com a qualidade da ordenação estocástica dos respondentes com relação ao seu traço latente, baseado no escore total dos itens selecionados. De modo geral, a literatura considera aceitáveis valores de $\mathrm{H}$ acima de 0,30 ; com $\mathrm{H}$ maior que 0,40 tem-se um escala forte e valores inferiores a 0,30 indicam uma escala fraca. Escalas fortes são desejáveis; o ordenamento dos respondentes pelo escore total observado é feito com maior segurança quanto maior o valor de $\mathrm{H}$.

O programa estatístico MSP (Mokken Scale for Politomous items) pode ser usado em modo exploratório, quando possíveis subconjuntos de itens (escalas) que apresentam a melhor escalonabilidade $(\mathrm{H})$ são sugeridos; ou em modo confirmatório, quando são estimados os coeficientes de ajuste para o conjunto de itens estipulado pelo pesquisador.

Com o intuito de avaliar a presença de viés implícito no escore total, ou seja, se existe diferença significativa entre a mediana do escore total nos subgrupos (categorias recodificadas das variáveis sociais descritas anteriormente), utilizamos o teste da mediana. Este procedimento estatístico não paramétrico envolve, essencialmente, um teste de significância baseado na distribuição qui-quadrado em uma tabulação cruzada, para determinar se há evidências de diferença significativa entre as medianas de dois ou mais subgrupos independentes. Para efetuar este teste, utilizamos o software SPSS- Statistical Package for the Social Sciences - versão 17.0.

\section{RESULTADOS}

A amostra foi constituída de $90 \%$ de mulheres; os casados compreendiam $42 \%$ da amostra, enquanto $33 \%$ declararam-se viúvos, $17 \%$ separados ou divorciados, $6 \%$ solteiros e $2 \%$ reportaram outro estado civil. Com relação à escolaridade, $60 \%$ dos idosos tinham ensino fundamental, $36 \%$ ensino médio e somente $4 \%$ nível superior. Metade dos respondentes tinha mais de 60 anos, $44 \%$ aproximadamente 70 anos e $6 \% 80$ anos ou mais.

\section{As escalas relacionadas à capacidade de autocui- dado de idosos}

A partir dos 24 itens do questionário ASA-A, o MSP, em seu modo exploratório, sugeriu duas escalas. A primeira incluindo 11 itens, com $\mathrm{H}=0,39$; a segunda com 8 itens, com $\mathrm{H}=0,37$, ambas indicando escalas razoáveis ${ }^{(9)}$ para itens do autocuidado do idoso. As escalas foram refinadas e assim ficaram interpretadas: uma como ações e atitudes subjetivas que o idoso realiza para manter-se saudável (11 itens listados na tabela 1) e a outra como ações e atitudes objetivas que o idoso realiza 
para manter-se saudável (8 itens listados na tabela 2).

Os coeficientes de escalonabilidade dos itens $\left(\mathrm{H}_{\mathrm{i}}\right)$ e da escala (H), bem como o coeficiente de confiabilidade da teoria não paramétrica (Rho de Mokken), foram calculados por este programa e estão também destacados nas referidas tabelas. Cinco itens do questionário ASA-A foram excluídos desta análise por não terem formado uma escala de Mokken, seja por apresentar correlação negativa com os demais ou por ter escalonabilidade muito baixa.

A partir dos dados da tabela 1, constata-se que: os itens de mais fácil concordância, isto é, mencionados em maior frequência pelos idosos foram os itens 8 (toma banho, sempre que necessário, para manter a sua higiene), 4 (procura manter limpo e saudável o lugar onde vive), 6 (sempre que pode cuida de si) e 5 (quando necessário, toma providências para manter-se saudável). O item mais difícil foi o item 16 .
Dos valores das médias e dos erros-padrão da média é possível distinguir três grupos de itens: o grupo de itens mais fáceis, que têm média maior ou igual que 4,5 (itens 8, 4, 6, 5, 7 e 1 acima de 'concordo'; o grupo de itens medianamente aceitos (itens 21,19 e 17) com média em torno de 4 ('concordo') e o grupo de itens mais difíceis (itens 15 e 16) com médias abaixo de 4 (abaixo do 'concordo').

A coluna com os coeficientes da escalonabilidade de cada item mostra a capacidade de discriminação do item. $\mathrm{O}$ item 21 (com $\mathrm{H}=0,46)$ tem alta capacidade de discriminação, ou seja, ele é capaz de distinguir mais fortemente o conportamento entre os idosos. Já o item $6(\mathrm{com} \mathrm{H}=0,28)$ tem pequena capacidade de diferenciar o comportamento dos idosos.

Da mesma, forma a tabela 2, mostra que o item de mais fácil concordância é o 10 (quando necessário, reserva um tempo para estar consigo mesmo) e o item mais difícil éo item 13 (geralmente, dorme o suficiente para se sentir descansado).

Tabela 1 - Resultados para Escala 1- Capacidade Subjetiva de Autocuidado. Niterói, 2008

\section{Escalonabilidade $\mathrm{H}=\mathbf{0 , 3 9}$; Confiabilidade $\mathrm{Rho}=\mathbf{0 , 8 2}$}

8 - Toma banho, sempre que necessário, para manter a sua higiene.

4 - Procura manter limpo e saudável o lugar onde vive.

6 - Sempre que pode, cuida de si.

5 - Quando necessário, toma providências para manter-se saudável.

7 - Procura as melhores maneiras de cuidar se si.

1 - Quando acontece qualquer tipo de alteração na sua vida, você procura fazer as mudanças necessárias para manter-se saudável.

21 - Se tem algum problema de saúde, sabe como conseguir informações para resolvê-lo.

19 - Costuma avaliar se as coisas que faz para manter-se saudável têm dado bom resultado.

17 - No passado, mudou alguns de seus antigos costumes para melhorar sua saúde.

15 - De tempos em tempos examina seu corpo para ver se há alguma diferença.

16 - Antes de tomar um remédio novo procura informar se ele causa algum mal-estar. média e-p da $\mathrm{H}_{\mathrm{i}}$ média

$4,85 \quad 0,00 \quad 0,38$

$\begin{array}{lll}4,71 & 0,08 & 0,35\end{array}$

$\begin{array}{lll}4,62 & 0,09 & 0,28\end{array}$

$\begin{array}{lll}4,56 & 0,08 & 0,36\end{array}$

$4,50 \quad 0,12 \quad 0,43$

$4,46 \quad 0,10 \quad 0,44$

$4,31 \quad 0,13 \quad 0,46$

$4,10 \quad 0,13 \quad 0,37$

$3,98 \quad 0,18 \quad 0,32$

$3,81 \quad 0,21 \quad 0,42$

$3,54 \quad 0,20 \quad 0,33$

Tabela 2 - Resultados para Escala 2- Capacidade Objetiva de Autocuidado. Niterói, 2008

\section{Escalonabilidade $\mathrm{H}=0,37$; Confiabilidade $\mathrm{Rho}=0,81$}

10 - Quando necessário, reserva um tempo para estar consigo mesmo.

12 - Com o passar dos anos, fez amigos com quem pode contar.

20 - No seu dia-a-dia, geralmente encontra tempo para cuidar de si mesmo.

2 - Geralmente vê se tudo aquilo que faz para manter sua saúde está certo.

23 - Sempre acha tempo para si mesmo.

24 - Mesmo tendo dificuldades para movimentar alguma parte do seu corpo, geralmente consegue cuidar-se como gostaria.

9 - Procura alimentar-se de maneira a manter seu peso certo.

13 - Geralmente, dorme o suficiente para se sentir descansado. média e-p da $\mathbf{H}_{\mathrm{i}}$ média

$\begin{array}{lll}4,21 & 0,13 & 0,36\end{array}$

$4,10 \quad 0,17 \quad 0,26$

$4,10 \quad 0,15 \quad 0,38$

$4,10 \quad 0,14 \quad 0,32$

$3,98 \quad 0,17 \quad 0,46$

$3,92 \quad 0,17 \quad 0,44$

$3,54 \quad 0,17 \quad 0,45$

$3,52 \quad 0,20 \quad 0,31$

Cogitare Enferm. 2011 Out/Dez; 16(4):661-6 
Observando os valores das médias e dos errospadrão da média é possível distinguir dois grupos de itens: o grupo de itens medianamente aceitos (itens 10, 12, 20, 2 e 23) com média em torno de 4 ('concordo'); e o grupo de itens menos aceitos (itens 24, 9 e 13), com média abaixo de 4 (abaixo do 'concordo').

De novo, a coluna com os coeficientes da escalonabilidade de cada item mostra a capacidade de discriminação do item. Os itens 23,9 e 24 (com $\mathrm{H}=0,46, \mathrm{H}=0,45$ e $\mathrm{H}=0,44$, respectivamente) têm alta capacidade de discriminação, ou seja, eles são capazes de distinguir mais fortemente o comportamento entre as pessoas. Todos os itens dessa escala têm poder razoável de discriminação; o índice mais baixo é verificado com o item 12 (com $\mathrm{H}=0,26)$.

A distribuição da medida de Capacidade Subjetiva de Autocuidado (o escore total relativo à Escala 1) apresenta assimetria negativa, assumindo valores 30; 48 e 55, respectivamente, para mínimo, mediana e máximo, o que indica um alto grau de concordância com as ações e atitudes subjetivas que o idoso endossa no sentido de manter-se saudável.

Já para o escore total da Escala 2, os valores para mínimo, mediana e máximo são 15; 32,5 e 40, respectivamente. Isso mostra que, quando se trata das ações e atitudes objetivas dos cuidados com a saúde, os idosos tendem a mostrar menor concordância.

O teste da mediana mostrou que não há evidências, ao nível de significância de 5\%, que as medidas construídas apresentem viés implícito. Este resultado pode indicar que as medidas de capacidade de autocuidado objetivo e subjetivo construídas podem ser usadas nos diversos subgrupos definidos, sem que sejam verificados desvios indesejáveis.

O valor de cada medida construída separadamente (as Capacidades Subjetivas e Objetivas de Autocuidado, através dos escores totais) é afetado pelas seguintes variáveis: sexo, idade, escolaridade e estado civil.

\section{DISCUSSÃO}

A existência de determinados espaços como programas de educação em saúde com idosos para informações sobre autocuidado, a atuação de enfermeiros e outros profissionais de saúde e a da mídia, pode favorecer o autocuidado, seja ele objetivo ou subjetivo $^{(10)}$. Os idosos desta pesquisa, apesar de estar em constante contato com os enfermeiros que realizam educação para a saúde no programa, podem apresentar diferenças com relação à capacidade de se envolver e executar as ações aprendidas, como as de autocuidado, levando em consideração fatores condicionantes.

Certas características das pessoas como idade, sexo, cultura, condições sociais, apoio familiar e outros, são essenciais na organização das informações no dia a dia.

As mulheres sofrem muito mais de condições crônicas incapacitantes, como artrite, o que aumenta a probabilidade de buscar mais cuidados de saúde que os homens, e se utilizam de mais serviços de atenção primária objetivando o seu autocuidado. Um fator determinante também é que os homens, geralmente, são cuidados em casa pelos familiares e só procuram ajuda de profissionais quando os cuidados domésticos já não são suficientes para manter a saúde satisfatória. De acordo com os dados apresentados e o número de mulheres idosas, nesta pesquisa, constatase que as elas são mais objetivas no seu autocuidado em relação aos homens.

As mulheres idosas, em relação ao cuidado subjetivo, demonstram pior escore. Percebe-se, por meio de estudo, que as mulheres mostram-se mais doentes, dependentes, sozinhas, pobres e queixosas do que os homens, além de apresentar expectativas mais baixas e ser menos valorizadas ${ }^{(11)}$.

A literatura aborda que o estado civil possui influência direta no cuidado do indivíduo, tanto subjetivo como objetivo, uma vez que o apoio da família é um dos fatores condicionantes do autocuidado ${ }^{(2)}$.

Sabe-se que a escolaridade é um fator que influencia no autocuidado, pois quanto maior a escolaridade maior a preocupação e a atenção com a saúde. O risco de se desenvolver problemas de saúde é inversamente proporcional ao número de anos de escolaridade formal, sendo que indivíduos com menos de 3 anos de escolaridade apresentam um maior índice de complicações do que aqueles com 11 anos ou mais de escolaridade ${ }^{(12)}$.

Com o aumento da expectativa de vida, o número de doenças crônicas e degenerativas tende a aumentar e sabe-se o quanto este fato pode trazer comprometimento da capacidade funcional e, consequentemente, do autocuidado tanto objetivo quanto o subjetivo do idoso. A idade interfere na capacidade, no tipo, e na quantidade de autocuidado requerido, seja ele subjetivo ou objetivo ${ }^{(13)}$.

Assim, os profissionais de saúde, principalmente o enfermeiro, devem estar atentos às diferenças e necessidade de apoio quando ocorre o déficit de autocuidado objetivo e/ou subjetivo no idoso.

\section{CONCLUSÕES}

Dos 24 itens analisados, 19 formaram duas escalas: 
(1) Capacidade Subjetiva de Autocuidado, mostrando a concordância com ações e atitudes subjetivas que o idoso realiza para manter-se saudável (com 11 itens e índice de escalonabilidade $\mathrm{H}$ de Löevinger, de 0,38); e (2) Capacidade Objetiva de Autocuidado, mostrando a concordância com ações e atitudes objetivas que o idoso realiza para manter-se saudável (com 8 itens e $\mathrm{H}=0,37$ ).

$\mathrm{Na}$ amostra estudada, verificou-se que quando se trata de ações e atitudes objetivas, a capacidade de autocuidado dos idosos é menor do que quando se trata de ações e atitudes subjetivas. Para os profissionais de saúde, deve-se ter em mente que os idosos valorizam ou buscam, com maior frequência, as ações e atividades de autocuidado subjetivas: tomar providências para manter-se saudável; fazer as mudanças necessárias para manter-se saudável; quando tem algum problema de saúde, sabe como conseguir informações para resolvê-lo; mudança de alguns de seus antigos costumes para melhorar sua saúde, e outros.

Finalmente, é imprescindível que nos programas universitários, principalmente os de Enfermagem, os docentes, alunos e profissionais, observem e enfatizem o autocuidado subjetivo, não desvalorizando o autocuidado objetivo, tendo em vista que estas ações são essenciais para a qualidade de vida do idoso.

\section{REFERÊNCIAS}

1. Instituto Brasileiro de Geografia e Estatística (IBGE). [Internet]. 2004 [acesso 05 jan 2008]. Disponível: http:// www.ibge.gov.br.

2. Foster PC, Benet AM, Dorothea Orem. Teorias de enfermagem: os fundamentos para a prática profissional. In: George JB. Teorias de enfermagem: os fundamentos para a prática profissional. $4^{\mathrm{a}} \mathrm{ed}$. Porto Alegre: Artmed, 2000.

3. Sá SPC. A representação social da velhice e as implicações do cuidado de si. [tese]. Rio de Janeiro (RJ): Universidade Federal do Rio de Janeiro; 2004.

4. Evers GCM. Appraisal of self-care agency ASA-scale. Assen Maastricht: Van Gorkum; 1989.

5. Silva JV, Kimura M. Adaptação cultural e validação do instrumento de medida de capacidade de autocuidado do "Appraisal of Self-care Agency Scale (ASA-A)". In: $13^{\circ}$ Seminário Nacional de Pesquisa em Enfermagem, 2005, São Luiz, Brasil. São Luiz: ABEn;2005.

6. Mokken RJ. A theory and procedure of scale analysis. The hague. Mouton: De Gruyter; 1971.
7. Molenaar IM, Sijtsma K. User's manual, MSP5 for Windows, ProGamma, Groningen; 2000.

8. Loehlin JC. Latent Variable Models: An introduction to factor, path, and structural equation analysis. 4th ed. Hillsdale: Erlbaum; 1987.

9. Sijtsma K, Molenaar IM. Introduction to nonparametric item response theory. New York: Sage; 2002.

10. Raudenbush SW, Sampson RJ. Seeing Disorder: neighborhood stigma and the social construction of "broken windows". Social Psychology Quarterly. 2004;67(4):319-42.

11. Neri AL. Qualidade de vida na velhice: enfoque multidisciplinar. Campinas: Alínea; 2007.

12. Almeida MF, Barata RB, Montero CV, Silva ZP. Prevalência de doenças crônicas auto-referidas e utilização de serviços de saúde, PNAD/1998, Brasil. Ciênc Saúde Coletiva. 2002;7(4):743-56.

13. Milhomem ACM, Mantelli FF, Lima GAV, Bachion MM. Diagnósticos de enfermagem identificados em pessoas com diabetes tipo 2 mediante abordagem baseada no modelo de Orem. Rev Eletron Enferm. [acesso em 12 de jan 2011]. 2008;10(2). Disponível: http://www.fen.ufg.br/revista/v10/n2/pdf/v10n2a04.pdf

Cogitare Enferm. 2011 Out/Dez; 16(4):661-6 